\title{
Olbers' Paradox Resolved for the Infinite Nonexpanding Universe
}

\section{Conrad Ranzan}

DSSU Research, Niagara Falls, Canada

\section{Email address:}

Ranzan@CellularUniverse.org

\section{To cite this article:}

Conrad Ranzan. Olbers' Paradox Resolved for the Infinite Nonexpanding Universe. American Journal of Astronomy and Astrophysics. Vol. 4, No. 1, 2016, pp. 1-14. doi: 10.11648/j.ajaa.20160401.11

\begin{abstract}
The conventional argument holds that the night sky is dark because the universe is expanding and the lifespan of stars is limited. Expansion weakens the light and star deaths reduce the number of light sources. Ultimately there will be no new formation of stars. However, the conventional resolution of Olbers' Paradox is no longer tenable. It turns out that the Universe is not expanding, the Universe is not evolving, and there is no restriction on the number of stars - the process of star formation is perpetual. With the recent publication of two revolutionary papers, one describing a simple cosmic redshift mechanism wherein wavelength elongation occurs in both expanding space and contracting space and the other detailing the resolution of what is undisputedly the most intractable anomaly in astronomy and astrophysics, the true nature of the Universe has been revealed - our world is a steady state cellular cosmos. The real Universe does not expand; furthermore, the numbers of light sources are infinite and their average spacing density never changes (since they are perpetually being replaced). Clearly, neither of the two conventional arguments will work. The following is a new two-part resolution of the night-sky-darkness question - a natural resolution that exploits the new cosmic redshift mechanism, known as the velocity-differential shift, and a photon extinction-probability mechanism.
\end{abstract}

Keywords: Olbers' Paradox, Cosmic Redshift, Photon Propagation, Cosmic Background Radiation, Gravity Cell, Dynamic Aether, Cellular Cosmology, Cosmic Structure, Gravity Domain, Big Bang, DSSU

\section{History of the Night-Sky Puzzle}

\subsection{Background}

The $15^{\text {th }}$ and $16^{\text {th }}$ centuries were a period in which there was a renewed interest in ancient knowledge, particularly secret knowledge and various newly-deciphered writings and inscriptions. In 1417 an unusual work by the Roman poet, Lucretius, was discovered. The manuscript, The Nature of the Universe, was an epic poem in praise of the Greek-conceived Epicurean universe of countless worlds distributed throughout infinite space. Lucretius outlined an infinite universe where "nature is free and uncontrolled by proud masters and runs the universe by herself without the aid of gods."[1] It may well have been history's first "scientific" universe.

Probably the most extraordinary of the documents that surfaced in the $15^{\text {th }}$ century was a Greek manuscript, consisting of seventeen 'books', brought from Macedonia to Florence to the Cosimo de' Medici. It was said to contain the secret wisdom of the ancient Egyptian sage whom the Greeks called
Hermes Trismegistus. Translated into Latin, by the Florentine philosopher Marsilio Ficino (1433-1499) in 1471, it came to be known as the Corpus Hermeticum, a source for all the various and powerfully effective Hermetic philosophies that followed. [2]

It was the Corpus Hermeticum that inspired Nicolaus Copernicus to make the heretical claim that the Sun, not the Earth, rules the center of the Solar system. On the first page of De Revolutionibus, published in 1543, Copernicus quotes the words of The Hermetica: "The Sun is the Visible God." [3] And within the text, Copernicus actually cited the ancient sage himself, Hermes Trismegistus, in support of the key idea. [4]

Thomas Digges was similarly inspired. In a book published in 1576, he graphically placed the Sun at the center, the planets, including the Earth, in orbits around it, and the stars extending infinitely into space - not fixed to a celestial sphere as Copernicus had compliantly believed in keeping with his religion. [5]

The last great philosopher and Hermetic Egyptologist of the $16^{\text {th }}$ century was Giordano Bruno (1548-1600). Bruno distilled 
the key lesson of the lost wisdom of the pharaohs: Learn Nature's secrets and attain understanding of the Universe. The secret was that the universe was infinite and the power that drives the universe was infinite. His official confession to the Church Inquisitors states: "I presuppose an infinite universe, a work of infinite Divine power, because I consider it unworthy of the Divine power, and Goodness to produce only this world when it could have created infinitely many worlds similar to our earth, which I understand along with Pythagoras, to be an orb similar to the moon, and the other planets and stars, inhabited worlds, the immeasurable number of which, in infinite space, forms an endless universe."[6] It cost him his life.

Into this intellectual backdrop, Johannes Kepler, in 1610, advanced a potent argument against the concept of an infinite universe. For what it's worth, we should note Kepler's unmistakable conflict of interest. He served as the imperial mathematician to the emperor of the Holy Roman Empire under which Bruno had been executed just 10 years earlier. In a letter Kepler sent to Galileo, he stated: "You do not hesitate to declare that there are visible over 10,000 stars. The more there are, and the more crowded they are, the stronger becomes my argument against the infinity of the universe." For if the universe stretched away endlessly, with stars like the Sun swarming everywhere, then the whole celestial vault would be as luminous as the disk of the Sun. [7]

In realizing that in an infinitely large universe the stars would collectively outshine the Sun and observing that the night sky is, on the contrary, obviously dark, Kepler gave origin to the famous dark-night-sky paradox.

\subsection{Early Resolutions}

Kepler believed that the universe was enclosed within a dark cosmic wall that formed an impenetrable boundary, and he was therefore able to explain why the sky at night is dark. His argument uncompromisingly clarified the options: accept either a cosmic edge and a dark night sky, or no cosmic edge and a blazing sky associated with an infinite universe. With this choice between two distasteful concepts, Kepler had led cosmology into accepting a finite universe.

The resolution was not to last. The final decade of the $1600 \mathrm{~s}$ saw the decline of Kepler's finite world and the rise of Newton's infinite universe. Newton (1642-1727) was probably aware of the paradox but was more concerned with a similar version, one with more immediate urgency to his theory of gravity. In the gravity version of the problem the combined effect of numerous distant gravitational forces should be enormous. Why isn't the Earth, for instance, being torn apart by these forces pulling from all directions? Newton resolved his gravity paradox by assuming that the infinite universe is homogenous, and reasoning that the star distribution must be spherically symmetrical, and concluding that therefore their gravitational forces cancel on the large scale. In effect, the forces sum to zero over a cosmic distance in any direction.

Evidently, the universe had to be infinite for the gravitational forces to cancel out. But the light received from stars in opposite directions does not cancel, rather, it adds up. The price of resolving the gravity problem is a return of the dark-sky paradox.

Edmund Halley (1656-1742), in the year 1720, put forth the argument that the light from remote stars was just too faint to be detectable. In spite of having infinite stars in an infinite Newtonian universe, the light from the remote stars, he believed, is too faint to be detected (even in collective or aggregate sense). [8] As many astronomers later pointed out, that solution won't work because two of nature's elementary laws cancel each other out. ... True enough the intensity of light decreases in proportion to the distance, however, the number of light sources, at a parametric distance, increases as the square of the distance. In terms of the cosmic shells shown in Fig. 1, the amount of light received from the shell of radius $r$ decreases as $1 / r^{2}$ but is completely compensated by the increase in the amount of light coming from the $r$-shell, by an increase proportional to $r^{2}$. In other words, the two effects cancel each other. They cancel so that from each thin cosmic shell we would receive the same amount of light. Thus, in an infinite universe, with an infinite number of concentric cosmic shells, it all adds up to an infinite amount of light.

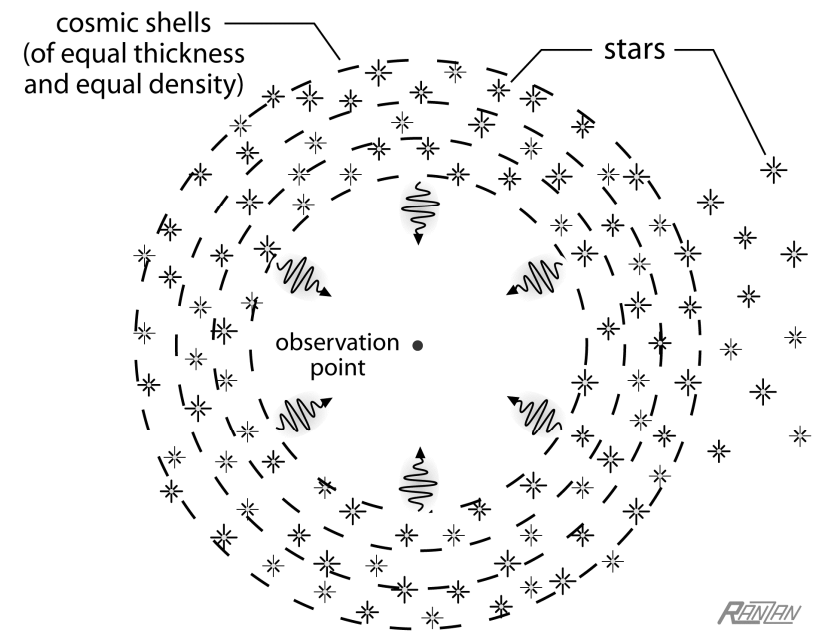

Figure 1. With stars distributed evenly throughout a three dimensional universe, the number of stars would be proportional to volume. Consider the concentric thin shells. The number of light sources in a shell increases with the radius, but the amount of light reaching the observer at the center decreases with the radius. The two effects cancel each other so that the same amount of light is received from each and every shell. In an infinite universe there are an infinite number of shells; thus, in the absence of any other effect, the observer would expect an infinite amount of light.

In 1744, the darkness of the night sky was discussed by a young Swiss astronomer, Jean-Philippe Loys de Cheseaux. He attributed the darkness to absorption of starlight by a fluid distributed throughout interstellar space. [8]

The German physician Heinrich Wilhelm Olbers (1758-1840), for whom the paradox became named, assumed that the presence of energy absorbing dust and other mass (like interstellar clouds) intercepted the starlight. The argument, proposed in 1823, seemed adequate at the time; but by 1848 its fatal flaw was pointed out. It was shown, by John Herschel, that the absorbing material must heat-up until it reaches an equilibrium temperature, at which the amount of energy it 
absorbs equals the energy re-radiated; and so the night sky would once again be blazing bright. The paradox needed some other explanation.

The American astronomer, Harlow Shapley (1885-1972), simply refused to accept the main premise of an infinity of stars. He along with a number of others in the early part of the $20^{\text {th }}$ century embraced the concept of the island universe - a finite world floating in an infinite dark void. There was, however, a complete lack of observational evidence; inevitably it failed. [9]

The discovery, during the $2^{\text {nd }}$ and $3^{\text {rd }}$ decades of the $20^{\text {th }}$ century, of the enormous extent of the universe of galaxies and the further discovery of the apparent systematic recession of those distant galaxies provided a new escape from the paradox. The expanding universe resolution. Representative of the early-period view was an argument advanced by Hermann Bondi, an advocate of the expanding universe, and focuses on the recessional motion of the stars: Because the universe is expanding, the galaxies, along with their stars, are all receding with the expanding space. Their light becomes weakened due to redshifting, with wavelengths being stretched beyond the visible range. If the universe is not expanding - if it were static - the sky would be exceedingly bright. Bondi's argument allowed the following choice: accept either an expanding universe (expanding because its space is expanding) and a dark night sky, or alternately, a static universe (associated with an unknown cause of redshifting) and a blazing sky. However, according to astrophysicist Edward Harrison, calculations have shown that redshift by itself is not sufficient. [10] A new resolution was needed.

At various times during the $20^{\text {th }}$ century it was proposed that the infinity assumption might be wrong; maybe the universe is actually finite and only appears limitless. Called the spherical space argument, it is an attempt to tame infinity and make the universe geometrically finite. It assumes an unbounded universe of finite size; and is analogous to the unbounded but finite area of a sphere. This removes Kepler's 'cosmic wall' and replaces it with the positive curvature of spherical geometry. In such a construction light rays traveling in any direction will eventually return to the starting point. Such is the nature of spherical geometry. Photons will repeatedly circumnavigate in this universe until striking some obstacle. However, geometric curvature is simply a mathematical concept; no one, it seems, can say what physical or real thing it is supposed to represent. It is merely an unworkable attempt to replace Kepler's brick wall with a mathematical construct. Depending on the details of the geometry, multiple images of each star may be predicted, flooding the universe with photons. The argument fails to resolve the paradox.

\subsection{Consensus Resolution}

The consensus of the experts of the late $20^{\text {th }}$ century settled on the finite time resolution. The cosmos, it was decided, must have a finite age. The resolution can best be explained in three parts: (i) The expanding universe is currently still too young and there has not been enough time for the light from distant stars to reach us. As the authoritative Encyclopedia
Britannica states, "Even if the universe is spatially infinite, photons from very distant galaxies simply [have not had] the time to travel to the Earth because of the finite speed of light." [11] (The concepts of a cosmos of finite time and of infinite extent do seem to be incompatible; but keep in mind that there is no consensus among the experts on whether the universe is spatially finite or infinite.) (ii) The lifetimes of stars are just too short; this means that the distant stars (somewhat closer than the ones in part one) now observable from Earth no longer exist. Those stars and star systems, their time having run out, are no longer contributing to the blazing sky problem. And (iii) there is a region outside our time zone - forever outside, forever invisible. Simply put, the consensus universe, the Big Bang, has an event horizon. This part of the resolution is called the cosmic event horizon resolution. As shown in Fig. 2, anything beyond this horizon is receding faster than the speed of light.

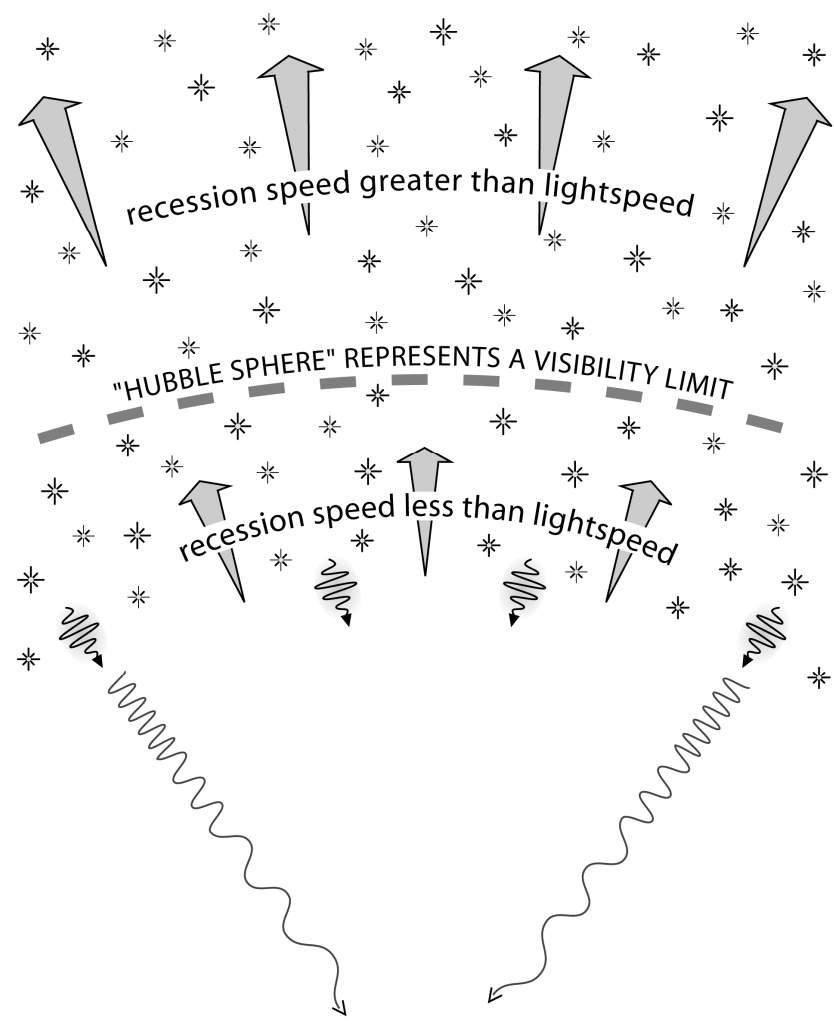

observation point

RANIAN

Figure 2. Expanding universe with expanding space has a visibility limit. The recession speed of stars located beyond what is known as the Hubble distance, or the Hubble sphere, exceeds the speed of light. This is because, for such a vast distance, the rate of expansion of the intervening space is greater than the speed of light. The Hubble sphere, for an observer located at the center, acts as a visibility limit and the light emanating from beyond is simply not observable, and never will become observable. It forms the edge of the observable universe censoring the infinite light from the surrounding infinite universe. The remaining stars, the ones within this visibility horizon, are just too few to produce a bright night sky. Thus, the Expanding universe is able to resolve Olbers' paradox.

There is one other resolution that is worth considering. It was proposed by physicist and astronomer Edward Harrison 
during the course of his many years of studying the dark-night-sky question. His energy-density resolution focuses on the simple premise that the universe was, at one time, blazing bright but has since gradually faded into darkness which we witness today (in the present era). The universe has, over time, cooled down.

The view is that at one time, during an early stage of Big Bang cosmogony, the sky was ablaze continuously. The universe was essentially a homogeneous 6000-degree gaseous "star." This star-universe expanded. As it expanded the temperature dropped. Over many billions of years, the isotropic expansion and decline in temperature continued until the night sky became dark. And at the present time, in this evolving universe, there is no dark-sky paradox because there is simply an insufficient energy density.

Given the condition described, of a hot-and-dense genesis, Harrison's argument is elegant and conclusive. Since the average density of all the matter in the present universe is extremely low - only one hydrogen atom per cubic meterthere is simply not enough equivalent energy. If all the existing mass were converted to pure energy, his calculations show that the thermal energy would equal only 20 degrees Kelvin -a radiation level that is certainly not in the visible spectrum and vanishingly far from the $6000 \mathrm{~K}$ temperature required by the bright-sky universe of the paradox. [12]

It would seem, then, that the paradox has been effectively resolved - at least for the expanding universe. However, the real Universe is not expanding.

\section{Need for a New Resolution}

\subsection{New Principle of Nature}

Harrison's argument is simple, elegant, and seemingly conclusive. The hot-and-bright universe became a cold-and-dark universe because it expanded. However, what if it happens that whenever a star expires another one takes its place? In other words, what happens in a perpetual steady-state universe in which radiation is not significantly recycled? In that case, unless some other factor or mediating influence is at work, the flood of photons being radiated into the universe would increase without limit. In this version of the paradox, stars are not only distributed throughout infinite space, but also shine (by endless renewal) throughout infinite time. And we are back to the prediction of a blazing sky — and again confronted with a paradox!

We will be examining an entirely new resolution of the paradox. We will do so specifically for a universe in which new stars are perpetually being formed and radiation is not, in any major way, recycled; and do so without violating global conservation of mass/energy.

But this is much more than a "what if" exercise. A number of recent developments have made it imperative.

The first one has to do with the fulfillment of an astute prediction made by Edwin Hubble — a prediction proffered during those early days when the interpretation of the cosmic redshift had not yet been settled. According to Allan Sandage [13]:
Hubble believed that his count data gave a more reasonable result concerning spatial curvature if the redshift correction was made assuming no recession. To the very end of his writings he maintained this position, favouring (or at the very least keeping open) the model where no true expansion exists, and therefore that the redshift "represents a hitherto unrecognized principle of nature."

That "hitherto unrecognized principle of nature" has now been found - more than three-quarters of a century after the discovery of the apparent recessional velocities of galaxies and many decades after the apparent recessional expansion was extrapolated into the expansion of the entire universe!

But let me back up a bit here. Some years ago, with the advent of $\mathrm{DSSU}^{1}$ cosmology came the realization that the universe is not expanding. The search was on for the proof. Rather fittingly, Edwin Hubble himself had provided an enigmatic clue with his "unrecognized principle of nature." The man accredited with discovering universe expansion did not believe that the universe was expanding. Hubble was convinced that the key evidence, the cosmic redshift, was caused by some other factor, something more fundamental than mere expansion.

With the recent discovery of a new cosmic-redshift mechanism, and its theoretical validation, it turns out that Hubble was right. [14]

\subsection{Game-Changing Discoveries}

Another clue came from Einstein. In his famous 1920 Leyden lecture, he had stated that the space medium (he called it ether) could not itself have mass or possess energy (he called it nonponderable). And, of course, the space medium had to be dynamic.

Probably the most profound development was the detailing of the fundamental process of energy [15], the process that effectively drives the DSSU.

By combining Einstein's key specifications with the DSSU's fundamental process of energy it was possible to construct a successful theory of gravity based entirely on space-medium dynamics [16]. This theory of gravity actually manages to combine contractile gravity, mass acquisition, and Lambda expansion, all under one unified concept.

The DSSU gravity theory made it clear that the universe is intrinsically cellularly structured and infinite in its three spatial dimensions. Most importantly for the present discussion is that it immediately led to the 'discovery' of a new redshift mechanism. But before we go into the details of how the space medium causes redshifting, I should clarify the status of the validity of DSSU cosmology.

The most recent development is the finding of irrefutable proof. Evidence in the form of a major long-standing observational anomaly clearly supports the validity of DSSU cos-

\footnotetext{
1 DSSU is the acronym for the Dynamic Steady State Universe - the cosmology theory that holds that the space medium is the ultimate bedrock of Nature, and further, that the space medium expands and contracts regionally and equally resulting in a cosmic-scale cellularly-structured universe. It is a model based on the premise that all things are processes.
} 
mology. The details are presented in the article: DSSU Validated by Redshift Theory and Structural Evidence [17]. There it is shown how the shape of the cosmic cells, as predicted by DSSU theory, are in amazing agreement with astronomically observed structures - structures quite inexplicable within the expanding-universe paradigm. It all leads to some profound cosmological implications; and yet, and this should raise eyebrows, the foundation premise of all modern cosmology is retained - retained but without extrapolation.

With the 'discovery' of a new redshift mechanism, the cosmology game had changed. It changed radically and permanently. As detailed in [14], the Expanding universe lost its main supporting pillar. Furthermore, with the unparalleled match between theory prediction and observed cosmic structure as presented in the DSSU Validation paper the concept of an expanding universe becomes untenable.

Recapping the game-changing developments: A new interpretation of Hubble's redshift; a successful aether theory of gravity; the resolution of what was, undoubtedly, the most intractable astronomical anomaly. When taken together, they constitute the establishment of a new cosmology —one with an unparalleled match between theory prediction and observed cosmic structure.

At the heart of the new cosmology is the velocity differential redshift mechanism. This simple process of light propagation leads to the conclusion that the Universe is not expanding.

Returning to Olbers' paradox, we impose the conditions demanded by a stable cellular cosmology (the DSSU) and ask the question: How is Olbers' paradox to be resolved if the Universe is infinite, Euclidean, perpetually regenerating, and non-expanding?

The night sky is dark, as E. Harrison had rightly argued, because the energy density of the universe is so low. So the question is this: Why then, given an infinite universe, an infinite number of stars and the perpetual replacement of those stars, is the energy density so extremely small? ... Based on these conditions why doesn't the energy content of the universe per unit volume just keep increasing?

There are two factors at play in ensuring the energy density compatible with night-sky darkness. One is the cosmic redshift; this will be discussed first. The other, to be discussed in Section 4 , is the probability of wave-packet capture and terminal annihilation. In the course of its cosmic journey, a photon faces the probability of such disruption; and this probability only increases with distance.

\section{First Factor: Energy Loss by Velocity-Differential Redshift}

There are a few things we need to understand about the propagation of light: (i) Light requires a conducting medium. (ii) A particle of light is a wave-like packet of excitation of the space medium. (iii) Light particles travel through gravity wells, often repeatedly, one gravity sink after another, unless captured. (iv) The space medium is always, everywhere, flowing; it is a dynamic flow. (v) Because a particle of light has a longitudinal dimension, its measurable wavelength, a velocity differential exists between its two extremities.

\subsection{Space Medium}

Light requires a conducting medium. As Einstein had said, without the presence of aether, there would be no propagation of light. In 1920 Albert Einstein stated as part of the Leyden lecture entitled Ether and the Theory of Relativity [18]:

"... there exists an [a] ether. According to the general theory of relativity space without [a] ether is unthinkable; for in such space there not only would be no propagation of light, but also no possibility of existence for standards of space and time (measuring-rods and clocks), nor therefore any space-time intervals in the physical sense."

But this aether should not be confused with any kind of mass or energy substance. In the same lecture, Einstein made it clear: "But this [a]ether may not be thought of as endowed with the quality characteristics of ponderable media ..."

Let me therefore emphasize, the space medium of DSSU theory, in its static state, has no energy and is not any form of mass. There is, however, one important difference: Einstein's space medium was a continuum, whereas DSSU's medium consists of discrete entities (non-material of course).

\subsection{Wave of Excitation}

Starlight can be thought of as a stream of photons. A photon is a wave-like excitation of the space medium. The nature of the excitation, the nature of the process by which photons are conducted by the aether, need not be detailed here. What is important is that the excitation has an axial distribution giving it a longitudinal dimension - giving the photon its characteristic measurable wavelength. The wavelength, of course, is what determines the energy of the light or the energy of its quantization as a photon. The relationship is inverse: the greater the wavelength, the lesser is the energy.

\subsection{Gravity Domains}

When starlight propagates through the universe it is always, and everywhere, traveling through gravity wells or domains. From the moment a bit of starlight is emitted, it is inside a gravity well. The packet of light first emerges from the star's own gravity well, then passes through its originating galaxy's gravity well, followed by a lengthy passage through the even larger galaxy-cluster gravity well. Unless interrupted, by being captured in some particle interaction, a wave packet will continue on its cosmic journey passing through one galaxy cluster after another -an endless series of cosmic-scale gravity domains.

The Universe consists of a dense packing of cosmic gravity domains each with a rich galaxy cluster at the center. See Fig. 3. In the figure, the domains are schematically modelled as spheres. In reality, however, the cosmos is tessellated into gravity domains that are shaped as tetrahedra and octahedral [19]. 


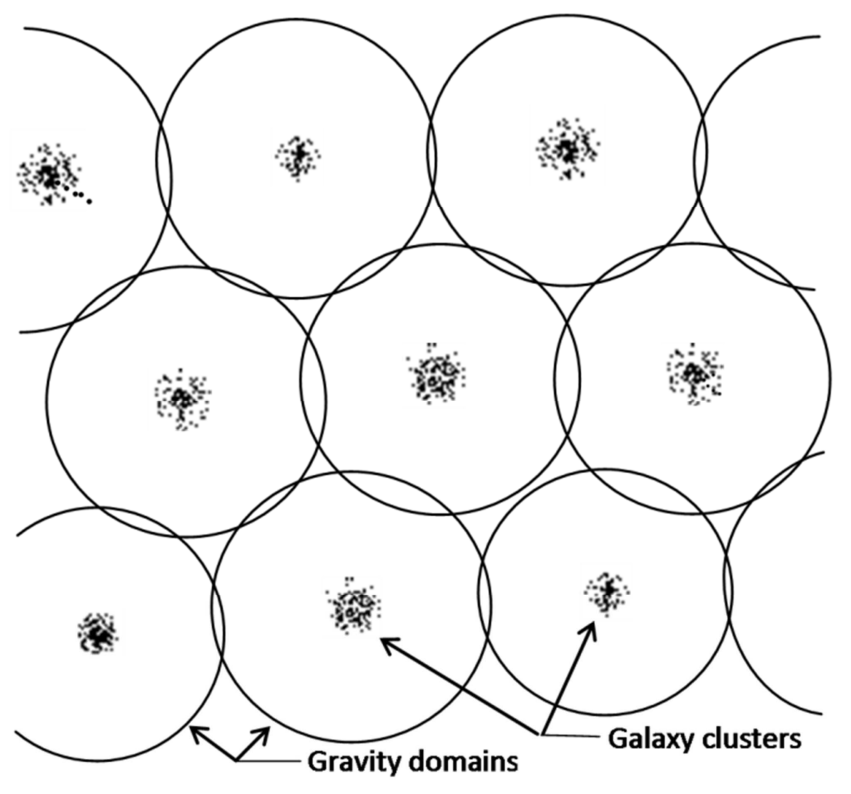

Figure 3. Cosmic gravity domains approximated, schematically, as spheres. The universe exists as a "packing" of gravity cells or domains, each with a rich galaxy cluster at its center. Consequently, starlight is always and everywhere subject to the dynamics within gravity domains. (Domains are about 200 to 350 million lightyears in diameter.)

\subsection{Flow of the Space Medium}

The gravity domains are entirely filled, or occupied, by aether (even within the interstices of the smallest atomic particles). Now, strange as it may seem, Einstein said that one cannot attribute motion to the aether [20], but he never explained why! Consider our perspective: DSSU aether is dynamic; it therefore possesses motion; it flows. According to the recently-validated aether-flow theory of gravity, all cosmic gravity domains involve an inflow of the space medium toward the center of gravity [16] [17]. A two-dimensional representation of the flow is shown in Fig. 4. The flow pattern defined by the cells is analogous to the way the height-of-land contours define the limits of a terrestrial watershed. In actuality, the internal flow within gravity cells is delineated by the surface boundaries of tetrahedra and octahedra; but the analogy with the height-of-land boundaries of a drainage-basin watershed is still conceptually useful.

If we place an axis through the center of a representative cluster, then a velocity profile will reveal that the flow of aether becomes increasingly negative (because the flow is in the negative direction of the radial-distance axis) as one moves nearer to the galaxy cluster. See Fig. 5. By far, the largest region of the domain is the medium-expansion region. Here there is an homologous type of expansion as reflected by the linearity of the velocity graph. The Expansion zone is where aether expands and the exponential growth in aether causes an acceleration of the aether toward the galaxy cluster. Simultaneously, the central region's Contractile zone is where aether contracts and as it contracts the remaining aether accelerates toward the core of the galaxy cluster.

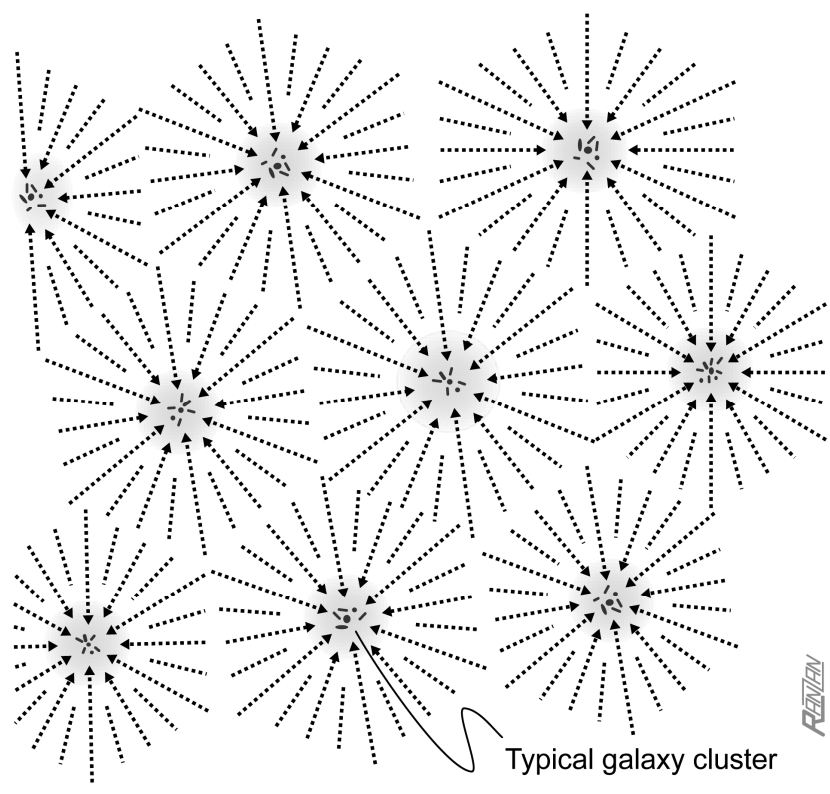

Figure 4. Flow of space medium within cosmic gravity domains. According to the DSSU aether theory of gravity, aether flows towards and into matter (aether thereby sustains the existence of matter). When starlight propagates through the universe it is propagating within aether that is always, and everywhere, in motion.

The graph tells us that, throughout the domain, aether (and anything comoving with the aether) is accelerating toward the center. It is, in fact, the acceleration that defines the domain as a gravity domain. Furthermore, it is a domain of limited extent; it has a "watershed" boundary; at the boundary the inflow velocity is clearly shown to be zero.

\subsection{Mechanism of Energy Loss}

Given that the photon is an extended particle by virtue of possessing a wavelength, requires a conducting medium by virtue of the fact that it has a fixed speed, and is at the mercy of that medium's motion by virtue of the photon being an excitation within (or of) that medium - the photon must consequently be subject to what is called the flow-differential effect. These factors provide the validation for the following analysis.

Let us consider a typical photon of starlight. And let us assume the photon is propagating along the direction of aether flow in a typical cosmic-scale gravity domain. Now, along the linear portion of the domain (Fig. 6), we already know that the photon will lose energy. Within a region of expanding vacuum, photons always lose energy. No one will ever dispute the prediction that wavelength elongation will occur in such a region (a region of space-medium expansion).

But what happens inside the region of space-medium contraction?

The photon passing through the contraction zone on its journey ever deeper into the gravity domain undergoes wavelength elongation. This is because there is a propagation velocity difference between the photon's two ends (as shown in Fig. 6). The front and back ends are actually moving apart. 


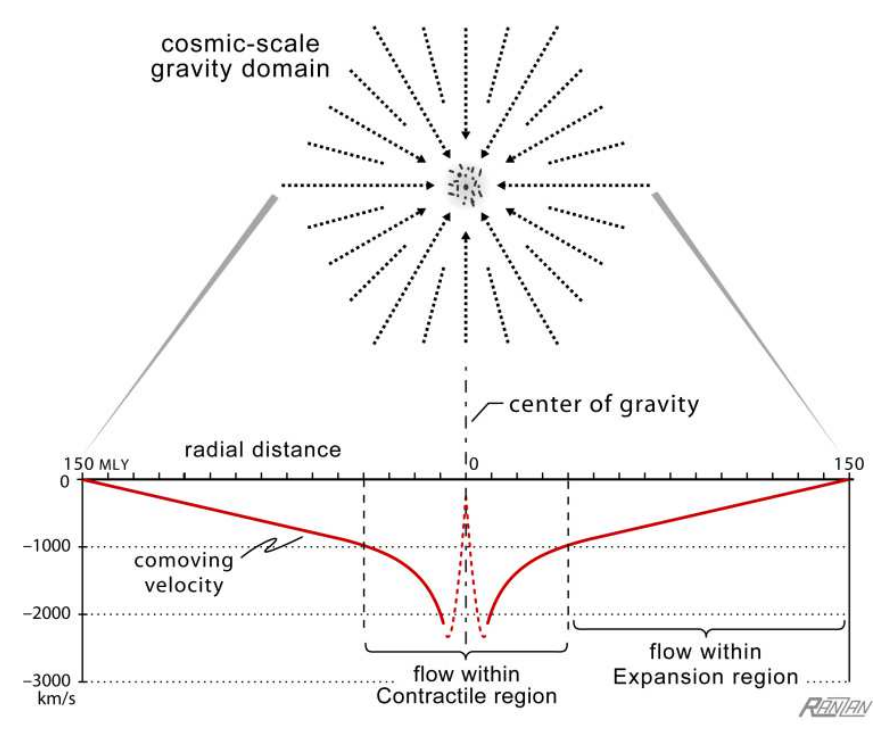

Figure 5. Velocity profile of the medium flow within cosmic gravity domain. The Contractile region is where aether contracts and the remaining aether accelerates toward the core of the galaxy cluster. The Expansion region is where aether expands and the exponential growth in the quantity of aether causes an acceleration of the aether toward the galaxy cluster. Note: It is not the regions that are expanding or contracting; it is only the space medium. For the most part, sizes of regions do not change and size of the domain does not change.

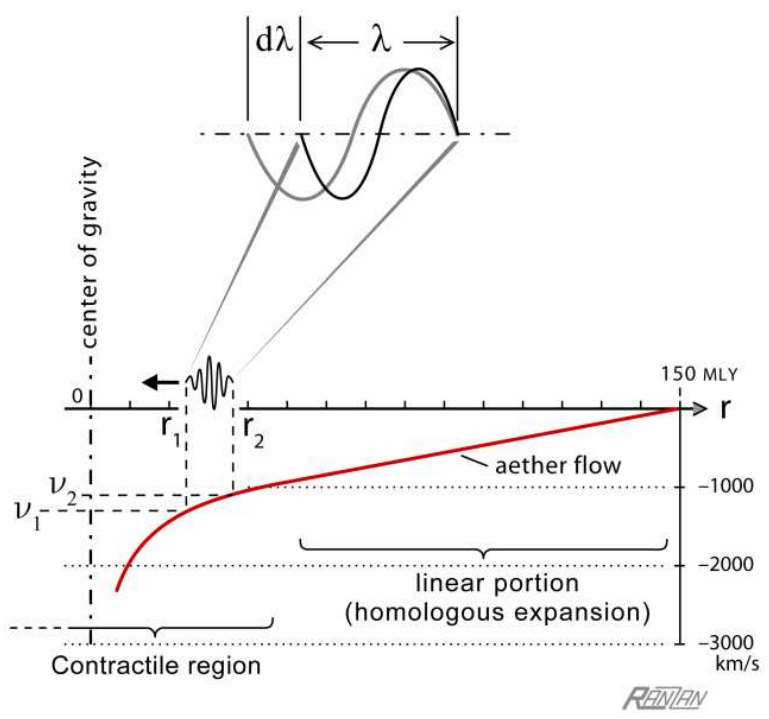

Figure 6. Photon elongation during inbound propagation through the Contractile region surrounding the galaxy cluster. The photon is being conducted by a space medium whose speed of inflow increases with proximity to the galaxy cluster (at least down to the limits of the cluster's "surface"). This same argument applies to the Expansion region, shown as the "linear portion" in the graph. As a result, the front and back ends of the photon "experience" a flow differential.

This can be shown mathematically. The speed of the front end of the photon is the speed of light $c$ plus the inflow speed of the aether $v_{1}$. The velocity of the front end of the photon is the negative of $\left(c+v_{1}\right)$. It is negative because it is in the opposite direction of increasing radius ( $r$-axis). Similarly, the velocity of the back end of the photon is the negative of $(c+$ $v_{2}$ ). The velocity-difference calculation is as follows:
(Relative velocity between ends of photon)

$$
\begin{aligned}
& =(\text { vel of front end })-(\text { vel of back end }) \\
& =\left[-\left(c+v_{1}\right)\right]-\left[-\left(c+v_{2}\right)\right] \\
& =\left(v_{2}-v_{1}\right)>0
\end{aligned}
$$

Since $v_{2}$ is higher on the velocity scale than $v_{1}$, the expression must be positive. (Or in simple terms, the front end of the inbound photon has a greater speed, in the direction of propagation, than does the tail end.) Hence, there is a velocity of separation between the two ends of the photon.

What is interesting is that this wavelength stretching occurs during the entire transit across a gravity domain. It occurs during the inbound as well as during the outbound segments.

For a photon emerging from, or coming out of, the depths of the gravity well (as shown in Fig. 7), the calculation is:

$$
\begin{aligned}
& =\left(c+v_{3}\right)-\left(c+v_{4}\right) \\
& =\left(v_{3}-v_{4}\right)>0 .
\end{aligned}
$$$$
(\text { vel difference })=(\text { vel front end })-(\text { vel back end })
$$

Since $v_{3}$ is more positive (that is, higher on the velocity scale) than $v_{4}$ (lower on the scale), the expression must be positive. Hence, again, there is a velocity of separation between the two ends of the photon.

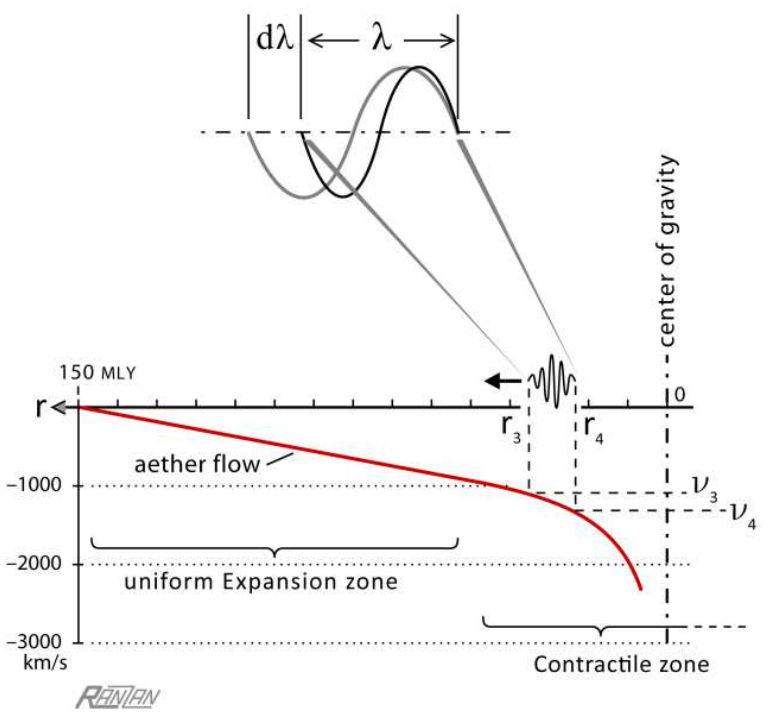

Figure 7. Photon elongation during outbound part of the journey across cosmic-scale gravity domain. The photon is being conducted by a space medium whose speed of inflow decreases with radial distance. (That is, the aether-velocity magnitude decreases until it reaches zero at the boundary where the radius is 150 million lightyears). As a result, in both the Expansion zone and the Contractile zone, the front and back ends of the photon "experience" a flow differential.

This moving-apart velocity between the two ends of the photon can be expressed as $d \lambda / d t$. Furthermore, it is proportional to the wavelength $\lambda$ itself. In equation form,

$$
\frac{d \lambda}{d t}=k \lambda
$$


where $k$ is the proportionality parameter, the fractional time-rate-of-change parameter, and

$$
k=\frac{1}{\lambda} \frac{d \lambda}{d t} .
$$

Now notice that $d \lambda / d t$ is simply the velocity difference between the photons two ends; and $\lambda$ is the difference between the front and back ends on the $r$-axis.

For the inbound photon in Fig. 6, the graph shows that $\lambda$ is $\left(r_{2}-r_{1}\right)$ and $d \lambda / d t$ is $\left(v_{2}-v_{1}\right)$. So that,

$$
k_{\text {inbound }}=\frac{\left(v_{2}-v_{1}\right)}{\left(r_{2}-r_{1}\right)} .
$$

For the outbound photon in Fig. 7 , the wavelength $\lambda$ is $\left(r_{3}-\right.$ $\left.r_{4}\right)$ and $d \lambda / d t$ is $\left(v_{3}-v_{4}\right)$ and,

$$
k_{\text {outbound }}=\frac{\left(v_{3}-v_{4}\right)}{\left(r_{3}-r_{4}\right)} .
$$

The parameter $k$ is simply the slope of the tangent to the velocity curve - and in both cases (inbound and outbound) $k$ represents a positive slope.

For the linear portions of the graphs, the slope $k$ is constant and it is reasonably easy to solve the differential equation (3) for $\lambda$ and determine the amount of wavelength elongation - the degree of weakening of the light.

For the curved portions of the gravity domain, $k$ is a function of the radial distance from the central galaxy cluster. And to find that function we start with the expression for approximating the aether-flow velocity (its simple derivation may be found in the article Cosmic Redshift in the Nonexpanding Cellular Universe: Velocity-Differential Theory of Cosmic Redshift [14]):

$$
v_{\text {aetherflow }}=-\sqrt{2 G M \mathrm{CL} / r},
$$

where $r \geq$ (radius of cluster "surface"), $G$ is the gravitational constant, and $M_{\mathrm{CL}}$ is the mass of the cluster.

The expression for the slope of the velocity curve is just the derivative,

$$
\frac{d v}{d r}=\frac{d}{d r}\left(-\sqrt{2 G M_{\mathrm{CL}} / r}\right)=\frac{1}{2} \sqrt{2 G M_{\mathrm{CL}}}\left(r^{-3 / 2}\right) .
$$

The slope $k$ may then be expressed for any radial location, $r$, as,

$$
k(r)=\frac{1}{2} \sqrt{2 G M_{\mathrm{CL}}}\left(r^{-3 / 2}\right) .
$$

With the substitution of (9) into (3), the $\lambda$ growth expression becomes,

$$
\frac{d \lambda}{d t}=\frac{1}{2} \sqrt{2 G M_{\mathrm{CL}}}\left(r^{-3 / 2}\right) \lambda
$$

or equivalently (by using the chain rule),

$$
d \lambda \frac{d r}{d t}=\frac{1}{2} \sqrt{2 G M_{\mathrm{CL}}}\left(r^{-3 / 2}\right) \lambda d r .
$$

But $d r / d t$ is just the speed of the photon itself, the speed of light $c$; and so

$$
\frac{d \lambda}{\lambda}=\frac{1}{2 c} \sqrt{2 G M_{\mathrm{CL}}}\left(r^{-3 / 2}\right) d r
$$

And it is this differential equation, which when solved for $\lambda$ in terms of $r$, determines the amount of wavelength elongation within the curved portion of the idealized gravity domain.

The solution requires an integration from some initial radius on the r-axis to some final radius:

$$
\int_{\lambda \text { initial }}^{\lambda} \frac{d \lambda}{\lambda}=\frac{1}{2 c} \sqrt{2 G M_{\mathrm{CL}}} \int_{r \text { initial }}^{r \text { final }}\left(r^{-3 / 2}\right) d r .
$$

When solved for the final wavelength, we obtain

$$
\lambda_{\mathrm{f}}=\left(\lambda_{\mathrm{i}}\right) \exp \left(\frac{1}{c} \sqrt{2 G M_{\mathrm{CL}}}\left(\left|r_{\text {initial }}^{-1 / 2}-r_{\text {final }}^{-1 / 2}\right|\right)\right) .
$$

Let me clarify the use of the absolute value for the last term in the exponent function: The absolute-value brackets are used to allow integration in both directions along the $r$-axis. This usage accommodates integration performed in the positive direction of increasing radius (for the outbound situation) as well as in the negative direction of decreasing radius (for the inbound situation). Alternately, if the integration were performed specifically for the inbound photon, the last term would come out as $\left(r_{\text {final }}^{-1 / 2}-r_{\text {initial }}^{-1 / 2}\right)$ and would be positive. Either way, and this is the important point, the "final" wavelength $\lambda_{\text {final }}$ will always be greater than $\lambda_{\text {initial }}$. (A more detailed solution appears in reference [17])

Thus, the light-weakening effect occurs in the expansion zone and in the contraction zone. But what about the intra-cluster region?

It should be noted that the velocity-differential redshifting also applies to the interior of each cluster. This is a region filled with large and small gravity wells - the overlapping gravity wells of all the individual galaxies and objects that comprise the cluster. The rule is: Whenever light traverses any gravity well, it acquires a velocity-differential redshift. And so, the process of light weakening continues within the interior of the galaxy cluster. As photons pass through those sub-domains, they continue to acquire velocity-differential redshift.

The main point of this section is that wherever the photon is along the $r$-axis and regardless of direction of propagation along the axis, the wavelength will increase. This, in turn, means that the z-index of the light will increase with cosmic distance. The light grows weaker.

One other point deserves mention. The accrual of redshift with light's transit across cosmic gravity domains is not merely of theoretical interest. Remarkably, the redshift-distance relationship that has been constructed based on 
the velocity-differential mechanism agrees with the redshift-versus-distance curve based on astronomical observations [14]. Moreover, the redshift mechanism and the agreement with observation-based cosmic-distance scale do not require any new physics.

Now, according to the conventional view, as explained by Edward Harrison, the cosmic redshift does not by itself resolve the paradox for the Expanding universe. Something more than expansion-weakened light is needed; and he claims that the limited lifetimes of stars is the more important determining factor [21]. (There is simply not enough time. Stars burn out long before the universe fills with stellar radiation.)

Similarly, the cosmic redshift does not by itself resolve the paradox for the steady-state cellular universe. Something more than velocity-differential redshift is needed; but it has nothing to do with stellar lifespans. There is another factor at play.

\section{Second Factor: Photon Capture Probability}

Now let us quantify the sources. Consider concentric shells, each having the same thickness but of ever greater radius (as in Fig. 8). We want to know how many photons are emitted from each shell. Or, equivalently, we want to determine how many radiating sources are present in each identifiable shell (identifiable by redshift).

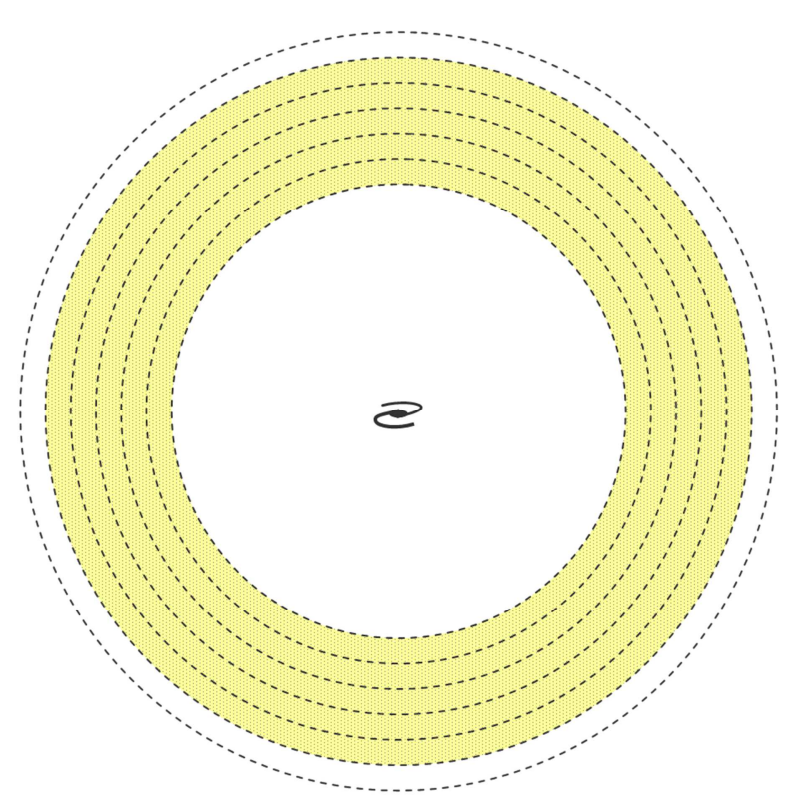

Figure 8. In a universe of uniform density, each larger shell has a greater number of light sources (stars) — simply by virtue of greater shell volume.

As discussed earlier, in a universe of uniform density and having no visibility horizon, each and every shell would be expected to contribute the same additional amount of photon flux. Each larger shell adds to the total flux. Since our universe is infinite in extent with an infinite number of concentric source-shells, then -in the absence of some other mechanism - the potential number of photons received would be infinite.

There is, however, a limiting factor in the form of an extinction mechanism. Before discussing photon extinction we must quantify the photon source.

\subsection{Number of Sources in Each Shell}

We have divided the universe into concentric spherical shells and have, thereby, constructed sort of an onion-like universe. A few of the shells are illustrated in Fig. 8. Each shell has a thickness, for convenience, of 300 million lightyears - a thickness corresponding to the nominal diameter of a typical dodecahedral-unit. Each shell contains vast numbers of photon sources: stars converting hydrogen into helium and emitting mostly yellow light.

The DSSU is homogenously cellular (Voids and galaxy clusters are more or less evenly distributed); thus the relative number of sources per "onion-layer" cosmic shell depends solely on the shell's volume. The volume of the first shell (actually a sphere) is $V_{1}=(4 / 3) \pi R^{3}$. Recall, we have chosen the shell thickness to be equal to the size of one dodecahedral-unit. Thus for the first shell the radius $R=1$ "unit" and $V_{1}$ $=(4 / 3) \pi$. The volume of the second shell, by simple calculation, is $V_{1}\left(2^{3}-1^{3}\right)$. The volume of the third shell is $V_{1}\left(3^{3}-2^{3}\right)$; and so on. The volume of the $N^{\text {th }}$ shell is $V_{1}\left(3 N^{2}-3 N+1\right)$. The volume of any shell is simply the volume of the first shell, $V_{1}$, multiplied by the factor $\left(3 N^{2}-3 N+1\right)$, where $N$ is the number of the shell.

Similarly, the number of sources in a particular shell is equal to the number of sources, $S_{1}$, in the first shell times the volume factor $\left(3 N^{2}-3 N+1\right)$. Thus, the number of sources in the $N^{\text {th }}$ shell is $S_{1}\left(3 N^{2}-3 N+1\right)$.

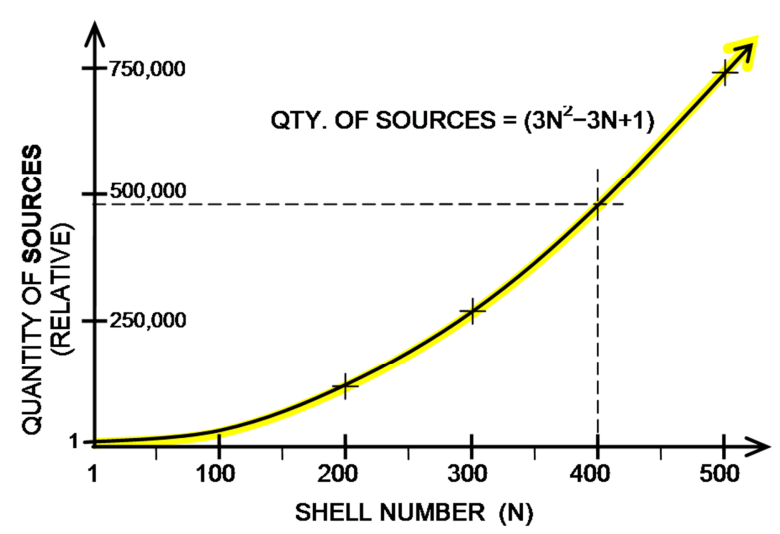

Figure 9. Function of relative number of typical light sources (stars) with respect to their corresponding originating shell. For example, the $400^{\text {th }}$ shell contains, within its thickness (between its inner and outer layers), 479,000 relative sources. Note that in the absence of a constraining factor the curve rises to the right without limit.

But since $S_{1}$ is constant, only the factor $\left(3 N^{2}-3 N+1\right)$ itself is needed to plot the relative number of sources corresponding to ever larger shells. Figure 9 shows the relative number-of-sources versus shell-number for 500 concentric shells. The actual number of sources could be obtained by, first of all, estimating a star count for the "first shell" and then multiply- 
ing this count times the relative source quantity (given by the graph) for the particular shell number of interest. (The numbers are surely stupendous.) The actual source count, however, is of little importance - the relative source count is what really interests us.

The graph above shows the relative photon count increasing without limit. In the real world this does not occur. The particles of light of the universe are subject to extinction. Extinction is the second phenomenon that affects a photon and ultimately the measurable flux. A photon, as it travels through successive cosmic shells (the onion layers of our analogy), may become captured and not make it across the thickness of one or another of those shells.

\subsection{Photon Extinction}

As a photon propagates through the universe it passes through one dodecahedral-unit after another. This naturally involves crossing the interface regions that separate voids. A photon may have a chance encounter, a chance interaction, with or without subsequently being re-emitted. For instance, it may be captured by a planet or a star. If it is captured and then re-emitted it loses its relationship with its original onion-layer shell -it effectively becomes a new photon, a new photon with no cosmic redshift. If it is not re-emitted then that photon, or its transformed manifestation, becomes permanently lost -i.e., it will eventually be terminally extinguished. Photons falling into terminal-state stars, for example, stand a good chance of being permanently lost. Another way of expressing the loss is by saying that the cosmic shells are imperfect black-body layers each having emissivity of 0.9950 - compared to a perfect black-body which has its emissivity equal to 1. Every time a photon travels through a shell (or through, or across, a single dodecahedral-unit) there exists a certain probability that it will not pass through. The estimate of the probability of such a loss is about 4 or 5 per thousand. Out of every 1000 photons, an average of 5 are lost during passage between consecutive shells. This $0.5 \%$ loss translates into an extinction factor of 0.0050 . It is this, and only this, factor that diminishes the photon-count from a cosmic source-shell.

Now considering the perspective from the receiving end. From our vantage point - our home galaxy - each more distant cosmic shell radiates vastly more photons, but to reach us they need to pass through an ever greater number of inner shells. During the transit through each shell thickness, $0.50 \%$ are lost, and $99.50 \%$ pass through. Of the photons originating within the first shell closest to us, a decimal fraction of 0.9950 , theoretically, passes through. Of the photons originating within the second shell, a fraction of $(0.995)^{2}$ makes it through; and so on, according to the rule of probability. Thus, for the photons originating from the $N^{\text {th }}$ shell and heading in our direction, a fraction of $(0.995)^{\mathrm{N}}$ succeeds in entering our Milky Way. It is easy to see that a large $N$-value makes for a low success rate. This tendency is illustrated in Fig. 10. Only about one-sixth of the photons originating in the $N_{400}$ shell actually make it to our local dodecahedral cell. (Only 13.5\% of the photons originating in the $\mathrm{N}_{400}$ cosmic shell reach our local dodecahedral cell. And, of course, the energy of each photon has decreased. The decrease is by a factor of 2850 , the same as the redshift factor, as we will see in a moment. Thermal energy has decreased by the same factor that wavelength has increased.)

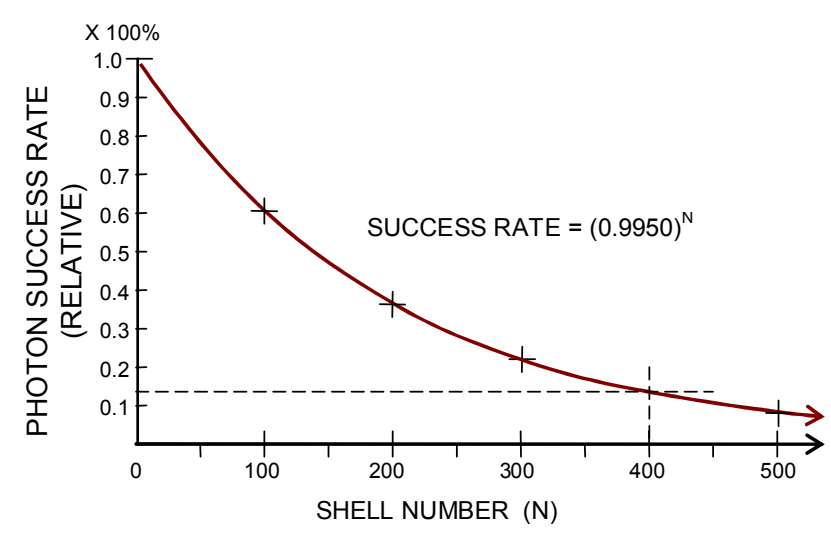

Figure 10. Curve gives the success rate of photons reaching the observation zone (at the center of concentricity). Each and every penetration of a shell reduces the photons' chance of reaching the next shell. For example, of the photons transmitted inward by the $400^{\text {th }}$ shell, only $13.5 \%$ will be detectable at the center.

\subsection{Intensity Variation}

So here is the situation: the deeper our Earth detectors peer into deep space, the more photons we expect to see (because there really are more sources), and simultaneously, the fewer photons we expect to see (because of the in-transit loss imposed by the extinction factor). We will now combine the two opposing tendencies and express the relative photon-count arriving at the center of all the concentric shells. The above analysis allows us to state that the detectable count, with respect to shell $N$, is

$$
(\text { Photon Count })_{\text {relative }}=(\text { Vol. Factor }) \times(\text { Success Rate })^{N}
$$

Notice that the Volume Factor diverges to infinity and the Success Rate term converges on zero. When the two are combined into a single function and plotted as in Fig. 11 the result is unequivocal; significantly, there is a clear peak in the intensity curve. The peak intensity corresponds to the region at or near the $400^{\text {th }}$ shell - a mind-boggling distance of 120 giga lightyears.

On the one hand, the number of photons emitted by consecutively larger shells increases geometrically in proportion to each shell's volume (which increases as the square of the radius). On the other hand, the number of photons that Earth detectors can pick-up diminishes with the distance in accordance with a probability factor that changes exponentially. In a competition between geometric growth and exponential change, it is the exponential change - in this case a decrease - that always wins in the end. Thus, as the distance to the emission source increases to infinity, the probability of being detected approaches zero. The curve in Fig. 11, if extended off the page, moves closer and closer to the horizontal 
axis and tends toward zero.

Let me emphasis the main point here. It is not so much the absolute intensity of the photon flux that is important; the measure of the absolute intensity is the responsibility of astronomers and is-what-it-is independent of one's theory. Nor is the relative intensity all that important; which, at best, is only an approximate prediction. The point of greatest consequence is the very existence of a maximum intensity - both in astronomical observations and in theoretical derivation - and its correspondence to a specific-and-unchanging distance. It manifests as a peak in cosmic radiation, the ubiquitous CBR; and it exists in a natural and self-evident theory.

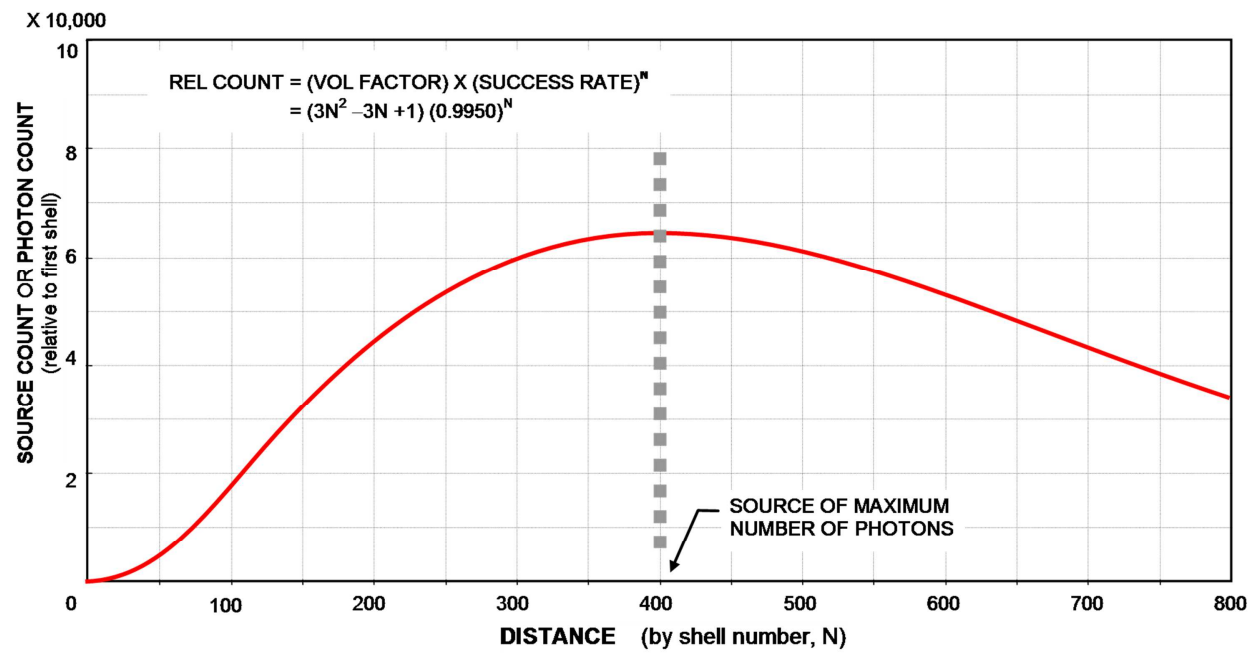

Figure 11. Theoretical relative photon-count of the radiation received from incremental distances by Earth detectors. For example, a space borne detector with a surface area of one square meter will receive twice as many photons from the $300^{\text {th }}$ shell as from the $140^{\text {th }}$ shell. Or for a truly dramatic comparison: for every one photon from the local shell impacting the detector there would be about 64,500 photons from the $400^{\text {th }}$ shell striking the detector.

\subsection{Aspects Relating to Extinction Factor}

The graph in Fig. 11 shows that the greatest abundance of photons comes to us from shell number 400 , that is, with the given extinction factor the prediction is that more photons will be received from there than from any other shell. Now let us relate this to what is actually observed.

The most abundant light sources in the Universe are, by far, red-hot to yellow-hot stars. It is a fact that $96.3 \%$ of the stars on the "main sequence" of the Hertzsprung-Russell (H-R) stellar classification system are within this range of 3000 to $6000 \mathrm{~K}$. In addition, there are a large number of high to extremely high luminosity stars above the main sequence of the $\mathrm{H}-\mathrm{R}$ chart. The great majority of these fall in the same temperature range and, hence, are classified as "red giants." Although the statistics of the H-R classification is based on the stars of the Milky Way galaxy, we accept, as a given, that the star-making process is the same throughout the Universe. Thus, the overwhelming source of radiation in the universe is from stars with surface temperatures of $3000 \mathrm{~K}$ to $6000 \mathrm{~K}$. The dominant photonic flow comes from red stars and yellow stars and everything in between. [22]

The emitted light, then, ranges from red (with a wavelength of about $750 \mathrm{~nm}=7.5 \times 10^{-7} \mathrm{~m}$ ) to yellow (with a wavelength of about $570 \mathrm{~nm}=5.7 \times 10^{-7} \mathrm{~m}$ ). The midpoint of this range is 660 $\mathrm{nm}=6.60 \times 10^{-7}$ meters. In other words the maximum intensity of the source radiation that dominates the Universe is a yellowish-red light of wavelength 660 nanometers.

This radiation scatters and permeates the Universe; it manifests as a chaotic background stream of photons. Subjected to numerous investigations by astronomers, the background radiation was found to have a maximum intensity at a wavelength of about 1.88 millimeters. Clearly what has happened is that the $6.60 \times 10^{-7} \mathrm{~m}$ light has had its wavelength stretched to $1.88 \times 10^{-3} \mathrm{~m}$ light, the consequence of a relentless cosmic redshift mechanism. Starlight that started out as $3000-6000^{\circ}$ temperature radiation has been weakened to a $3^{\circ}$ temperature. The redshift factor, $z$, responsible for the change is easy to calculate from its definition:

$$
\begin{gathered}
\text { Redshift }=\frac{(\text { observed wavelength })-(\text { emitted wavelength })}{(\text { emitted wavelength })} \\
z=\frac{\lambda \text { measured }-\lambda \text { source }}{\lambda \text { source }}=\frac{\lambda \text { measured }}{\lambda \text { source }}-1, \\
z_{\mathrm{CBR}}=\frac{1.88 \times 10^{-3}}{6.60 \times 10^{-7}}-1=2848-1 \cong 2850 .
\end{gathered}
$$

The source starlight has been redshifted by a factor of 2850 to become the measurable background radiation.

The equation for distance in the cellular universe, expressed in terms of the number of cosmic cells, is [23]:

$$
N(z)=\frac{\ln (1+z)}{\ln \left(1+z_{\mathrm{cc}}\right)},
$$

where $\mathrm{z}$ is the measured redshift, which in our case is $z_{\mathrm{CBR}}$ and equals 2850 . The symbol $z_{\mathrm{cc}}$ is the empirical redshift across one cosmic cell and, therefore, also across the thickness of one 
onion-layer shell. Its value is 0.020 per reference [17]. The distance then is:

$$
N_{\text {PeakSource }}=\frac{\ln (1+2850)}{\ln (1+0.020)}=\frac{7.954}{0.01980}=402 .
$$

Thus the source of the greatest abundance of photons comes from around the $400^{\text {th }}$ cosmic shell —a staggering distance of $120 \times 10^{9}$ lightyears (based on a shell thickness of $300 \mathrm{Mly}$ ).

Our calculated result agrees with the peak shown in the Fig.-10 graph; this should not be surprising since it was the distance calculated here that demanded the particular value chosen for the extinction factor. The extinction factor is the parameter used to make the graph fit the calculations (whose validity is based on measured quantities).

The extinction factor is affected by terminal-state stars; if there were no such stars then all radiation would eventually be re-emitted. The number of photons in the universe would forever increase! These special stars prevent this. So, what is meant by a terminal-state star?

Let me briefly explain how it happens that some photons are never re-emitted and instead become permanently lost. As stated above, a photon may be captured by a terminal-state star. This is a star in a critical state of extreme concentration of matter. It may be defined as a Dark Star (dark, because extreme gravity severely restricts the escape of radiation) having a core in which terminal-annihilation takes place if and when additional matter falls onto the surface of the star. Imagine such a star gaining mass; say an asteroid-size object were to fall in; at the instant that the star's mass increases by the mass of the asteroid, an equivalent amount of mass/energy will terminally annihilate at the core. Whenever mass and energy is captured, an equivalent amount thereof disappears at the terminal-process star's core. Matter literally disappears from the universe. The termination process only takes place in critical-state Dark Stars.

An obvious question is, Why not just make the claim that photons may fall into a black hole, a hypothetical object from which there is no escape? That would certainly terminate a lot of photons. Unfortunately (or rather, fortunately) black holes do not exist in the DSSU. They exist only as mathematical concoctions and employed by many physicists who have failed to understand the reality-based nature of gravitational collapse.

The terminal-state star and the role it plays in gravitational collapse, as well as night-sky darkness, is not an ad hoc concept but rather an integral part of the DSSU theory of matter and gravity.

These Dark Stars are key components responsible for maintaining a remarkable energy balance within cosmic cells (and, by extension, within our imaginary cosmic shells). They act as matter-limiting bodies. Any amount of mass or energy added to a non-rotating Dark Star will have no net affect on the star's mass/energy content. But this is only part of a more encompassing self-balancing mechanism, which involves an ongoing balance between the formation of matter and the termination of matter on a cosmic scale. What this means in the context of Olbers' paradox is that any external input of matter simply contributes to the rate of matter termination and the rate of photon re-emission occurring in a cosmic shell. On average, the energy density of any cosmic shell is the same; and the energy output (photon emission and re-emission) is sustained at a steady state rate. (Although, it should be noted, for any cosmic shell the net quantity of energy output and input is zero.) Of course, the great majority of external-sourced photons simply pass right through, never interacting with anything in a particular shell.

\subsection{Photon Extinction Summary}

There are two key factors affecting the photon count, the reduction (extinction) of incoming photons: (i) A photon may be captured (become part of an electron, say) but then its energy may be re-emitted. Because of a self-balancing mechanism between the formation of matter and the termination of matter, any external input of matter is of little consequence to the energy output of a cosmic shell. A perpetual steady-state equilibrium exists. If, as may only be imagined, an extraordinary amount of energy were to flow into a cosmic shell, then more energy would be re-emitted (and also more energy would undergo terminal extinction) until a steady state balance is restored. Any re-emitted photon is naturally considered to be a new photon with a new identity; and, importantly, it would possess no cosmic redshift. (ii) A photon may be captured and it, or its energy, is never re-emitted. This is called terminal annihilation. It is most likely to occur when a photon encounters the extreme gravitational environ of a neutron star.

The point is that proportionally fewer and fewer photons arrive from ever greater distances.

\section{Implications and Conclusion}

\subsection{New vs Old}

The traditional resolution of the night-sky paradox was to simply deny the infinity of sources and to naïvely claim that cosmic light is dimmed by the expansion of the universe. Both conditions were rejected; they did not apply to the new DSSU cosmology. What made the new resolution by far the most challenging is that the number of sources really is infinite; moreover, the universe is not expanding.

The resolution of Olbers' paradox, as we have seen, uses two perfectly natural arguments, both expressible in terms of mathematical functions. One argument was the exponential reduction in the photon count with respect to distance; and, the other, the exponential reduction in the photon energy by the cosmic redshift, likewise, with respect to distance. Both are relentless exponential factors. No wonder the night sky is dark.

The implications for the viability of universe expansion and of whole-universe evolution are surely the most profound aspects of the new resolution. The explanation of Olbers' paradox does not, in any way, require an expanding universe! Why demand that the universe perform the metaphysical trick 
of expanding itself to account for the darkness of the heavens? Why limit the number and duration of sources? As has been shown, a steady state cellular universe furnishes the definitive explanation and does so quite naturally —without cumbersome parameters, without supernatural processes.

The new resolution is dependent on autonomous gravity regions (cells) and a flowing medium (aether). Consequently, the resolution is incompatible with a General Relativity universe. The fact is that the General Relativity universe is not divided into autonomous gravity domains; rather, it is treated as a single gravity domain, in which the range of gravity has no limits and every object exerts a gravitational "pull" on every other object in the universe. In a sense, it is a single-cell universe, albeit with no center and no physical boundary (although it does have a mathematical boundary). However, the new resolution, based as it is on the velocity-differential redshift mechanism, requires a multiple-cell universe - having cells with definable boundaries. The new resolution demands the existence of multiple autonomous gravity domains - each with its own center of gravity. ... Even more important is the fact that General Relativity does not employ a flowing space medium. In Einstein's words: "We may assume the existence of an [a]ether; only we must give up ascribing a definite state of motion to it." And emphasized, "The [a]ether of the general theory of relativity is a medium which is itself devoid of all ... kinematical qualities." [24] In the absence of a dynamically flowing light-conducting aether, there would be no velocity variation and no meaning attachable to our "velocity-differential redshift."

Another point of comparison can be made here. Within the General Relativity paradigm, the expansion of space is the acknowledged factor responsible for the cosmic redshift, the weakening of light; but the contraction-of-space effect around gravitating bodies/regions is ignored as a contributing factor. Now consider how the Cellular paradigm extends our understanding. The DSSU cosmology, like all modern models, acknowledges "space" expansion as being responsible for the weakening of light. It then goes beyond this limitation and becomes the only cosmology to recognize that the contraction of space also shares responsibility for the weakening of light.

\subsection{Fatal Flaws}

The most successful cosmology in history, in terms of longevity, was the Ptolemaic System. But it had a fatal flaw. Its prediction of the phases of Venus were wrong; it was incapable of explaining the actual phases of the nearest planet to Earth.

The most successful, the most intricately detailed, the most highly acclaimed, cosmology in history, has a fatal flaw. The Big Bang model is hopelessly incapable of explaining the Abell-85 anomaly; it cannot, in any way, account for the startling regularity in the spacing of galaxy clusters strung out along the line-of-sight through Abell 85.

The Ptolemaic System's crippling defect remained hidden for many long centuries —until Galileo's important telescopic discovery of Venus's complete range of phases. Once the flaw was convincingly revealed, Geocentric proponents quietly went about changing their worldview. In contrast, the Big
Bang's flaw is glaringly out in the open and has been for decades. Whereas the experts should be abandoning the Big Bang model, or at least paving the way for a transition, they instead continue expressing commitment to a hypothesis for which they claim ever higher levels of confidence. This is a truly bizarre situation; it is intolerably embarrassing. It would be comparable to Galileo's contemporary experts continuing to defend and praise the Earth-centered system! Meanwhile, there are interested-and-informed spectators asking Why is it so easy to show that the Big Bang has failed - and has failed repeatedly? Why? And as of 2014 there is another factor to consider; there is now a realization that light waves stretch in both expanding and contracting regions of a gravity well, as detailed in reference [14]. It means, the Big Bang employs an incomplete interpretation of the cosmic redshift. It has missed the important unifying half of the redshift interpretation - thus rendering the expanding-universe hypothesis untenable and turning the Big Bang into a failed scenario. And so, the redshift interpretation is the heart of conventional cosmology's fatal flaw. (For the definitive proof see reference [17].)

And then there is DSSU cosmology, which is, by far, the most powerful problem-free system ever constructed. It thrives on seemingly intractable problems. It resolves the anomaly that defeated the Big Bang, and was the first cosmology to incorporate a unified cosmic redshift. The DSSU is a cellularly structured universe - intrinsically cellular. With its Euclidean cellular arrangement and its dynamic space medium, it readily explains the A85 anomaly; and, in fact, it predicts the type of pattern observed. Remarkably, although it is an infinite non-expanding perpetually-regenerating Universe, it predicts a dark night sky.

\section{References}

[1] E. R. Harrison, "Chap. 4 The Geometric Universe," in Masks of the Universe, Changing Ideas on the Nature of the Cosmos $\left(2^{\text {nd }}\right.$ ed. Cambridge University Press, Cambridge, UK, 2003) $p$ 54-55.

[2] P. Tompkins, "Chap. 3 Symbols of Heresy," in The Magic of Obelisks (Harper \& Row, Publishers, Inc.; New York; 1981) p $50-51$.

[3] T. Freke and P. Gandy, "Introduction," in The Hermetica, The Lost Wisdom of the Pharaohs (Penguin Putnam Inc., New York, 1997) p 14.

[4] Encyclopedia Britannica, "The History of Science," Vol. 27, 15 Edition (Encyclopedia Britannica, Inc., Chicago, 1991) p 37.

[5] C. Ronan, "Chap.7 From Renaissance to Scientific Revolution," in Science: its History and Development Among the World Cultures (The Hamlyn Publishing Group Ltd, New York, 1982) p 330 .

[6] P. Tompkins, "Chap. 4 Elizabethan Wits and Wizards," The Magic of Obelisks (Harper \& Row, Publishers, Inc.; New York; 1981) p 77-78.

[7] E. R. Harrison, "Chap. 12 Darkness at Night," in Cosmology, the Science of the Universe (Cambridge University Press, Cambridge, UK, 1981) p 250. 
[8] E. R. Harrison, "Chap. 12 Darkness at Night," in Cosmology, the Science of the Universe (Cambridge University Press, Cambridge, UK, 1981) p 251.

[9] E. R. Harrison, "Chap. 12 Darkness at Night," in Cosmology, the Science of the Universe (Cambridge University Press, Cambridge, UK, 1981) p 254.

[10] E. R. Harrison, "Chap. 12 Darkness at Night," in Cosmology, the Science of the Universe (Cambridge University Press, Cambridge, UK, 1981) p 263.

[11] Encyclopedia Britannica, "Cosmological Models," Vol. 16, 15 Edition (Encyclopedia Britannica, Inc., Chicago, 1991) p 787.

[12] E. R. Harrison, "Chap. 12 Darkness at Night," in Cosmology, the Science of the Universe (Cambridge University Press, Cambridge, UK, 1981) p 255.

[13] Allan Sandage, "Edwin Hubble 1889-1953," The Journal of the Royal Astronomical Society of Canada Vol. 83, No. 6. 1989, (http://antwrp.gsfc.nasa.gov/diamond_jubilee/1996/sandage_h ubble.html) (Retrieved March 26, 2010).

[14] C. Ranzan, "Cosmic Redshift in the Nonexpanding Cellular Universe: Velocity-Differential Theory of Cosmic Redshift," American Journal of Astronomy \& Astrophysics (AJAA). Vol .2, No. 5, 2014, pp. 47-60. (Doi: http://dx.doi.org/10.11648/j.ajaa.20140205.11).

[15] C. Ranzan, "The Fundamental Process of Energy -A Qualitative Unification of Energy, Mass, and Gravity, Part I": Infinite $\begin{array}{llll}\text { Energy Issue } \# 113 \quad \mathrm{Jan} / \mathrm{Feb} & 2014\end{array}$ (http://www.infinite-energy.com/iemagazine/issue113/index.ht ml). “... Part II”: Infinite Energy Issue \#114 Mar/Apr 2014 (http://www.infinite-energy.com/iemagazine/issue114/index.ht ml) (Posted at: www.cellularuniverse.org/).

[16] C. Ranzan, "The Processes of Gravitation -The Cause and Mechanism of Gravitation," Journal of Modern Physics and
Applications, Vol. 2014: 3 (2014). Posted at (http://www.cellularuniverse.org/G6GravityProcesses-abst\&.h tm).

[17] C. Ranzan, "DSSU Validated by Redshift Theory and Structural Evidence," Physics Essays Vol. 28, No. 4, p 455-473 (2015 Dec). (Doi: http://dx.doi.org/10.4006/0836-1398-28.4.455).

[18] A. Einstein, Sidelights on Relativity, translated by: G. B. Jeffery and W. Perret, Methuen \& Co. London, (1922) p 23.

[19] C. Ranzan, "The Dynamic Steady State Universe," Physics Essays Vol. 27, No. 2, pp. 286-315 (2014) (Doi: http://dx.doi.org/10.4006/0836-1398-27.2.286) (Posted at: www.cellularuniverse.org/).

[20] A. Einstein, Sidelights on Relativity, translated by: G. B. Jeffery and W. Perret, Methuen \& Co. London, (1922) p 24.

[21] E. R. Harrison, "Chap. 12 Darkness at Night," in Cosmology, the Science of the Universe (Cambridge University Press, Cambridge, UK, 1981) p 258-259.

[22] G. LeDrew, "The Real Starry Sky," Journal of the Royal Astronomical Society of Canada, Vol. 95, No. 1 (February 2001), pp $32-33$. (http://adsabs.harvard.edu/abs/2001JRASC..95...32L).

[23] C. Ranzan, "Cosmic-Redshift Distance Law Without c Without H," Galilean Electrodynamics Vol. 25, No. 3, pp. 43-55 (May/June 2014). (Posted at: www.CellularUnivese.org/D2CosmicDistEq.htm).

[24] A. Einstein, Sidelights on Relativity, translated by: G. B. Jeffery and W. Perret (Methuen \& Co. Ltd., 36 Essex St. W. C., London, 1922) p $13 \&$ p 19. 